\title{
MODELO COMPUTACIONAL PARA DETERMINAR EL NIVEL ÓPTIMO DE CARGABILIDAD DE LOS TRANSFORMADORES DE POTENCIA DEL SISTEMA NACIONAL INTERCONECTADO
}

\author{
Juan C. Rodríguez ${ }^{1, *}$ y Víctor H. Orejuela ${ }^{2}$
}

\section{Resumen}

En este documento se indica el proceso de desarrollo e implementación de un modelo computacional para determinar el nivel óptimo de cargabilidad de los transformadores de potencia del Sistema Nacional Interconectado del Ecuador. Gracias a las herramientas de las plataformas de software que permiten construir modelos matemáticos para hacer predicciones y optimizar el comportamiento de sistemas complejos, es posible desarrollar un modelo computacional que abarque todos los aspectos técnicos indicados y documentados en la normativa ANSI e IEC, para determinar los parámetros que inciden en la cargabilidad de los transformadores de potencia y las repercusiones que esto tiene en la vida útil de los transformadores. Bajo estos parámetros el modelo computacional, determina una solución; tanto desde el punto de vista técnico, como del económico; ya que al desarrollar este modelo en base a la formulación técnica-económica; se determina la cargabilidad óptima de los transformadores; y se establece una proyección de la vida útil del transformador de potencia dentro de la red de suministro eléctrico.

Palabras clave: ANSI (Instituto Nacional Americano de Normas), formulación técnico-económica, IEC (Comisión Electrotécnica Internacional), modelo computacional, nivel óptimo de cargabilidad.

\section{Abstract}

This document outlines the process of development and implementation of a computational model to determine the optimal level chargeability power transformers National Interconnected System of Ecuador. Thanks to the tools of software platforms that allow constructing mathematical models to make predictions and optimize the behavior of complex systems, it is possible to develop a computational model that encompasses all technical aspects indicated and documented in the ANSI and IEC, to determine the parameters affecting the chargeability of power transformers and the impact this has on the life of transformers. Under these parameters the computational model, determine a solution, both from a technical standpoint, and economically, and that in developing this model based on the technical development - economic, determining the optimal chargeability transformers, and establishes a projected life of the power transformer within the power grid.

Keywords: ANSI (American National Standards Institute), technical-economic development, IEC (International Electrotechnical Commission), computational model, chargeability optimal level.

\footnotetext{
$\overline{1, *}$ Estudiante de Ingeniería Eléctrica de la Universidad Politécnica Salesiana Sede Quito - Campus Kennedy. Autor

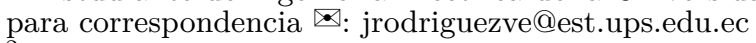

${ }^{2}$ Máster en Educación, Especialista en Seguridad y Desarrollo, Ingeniero eléctrico, Docente-Investigador de Ingeniería Eléctrica de la Universidad Politécnica Salesiana Quito - Campus Kennedy. Autor para correspondencia ${ }^{-}$vorejuela@ ups.edu.ec
}

Recibido: 17-05-2013, Aprobado tras revisión: 28-05-2013.

Forma sugerida de citación: Rodríguez, J. y Orejuela, V. (2013). "Modelo computacional para determinar el nivel óptimo de cargabilidad de los transformadores de potencia del sistema nacional interconectado". INGENIUS. N. ${ }^{\circ}$, (Enero-Junio). pp. 36-41. ISSN: 1390-650X. 


\section{Introducción}

\subsection{Antecedentes sector eléctrico ecuatoriano}

El constante aumento del número de usuarios y su correspondiente incremento de la demanda, necesita ser abastecido a través del Sistema Nacional de Transmisión - (SNT) que forma parte del Sistema Nacional Interconectado - (SNI). Esto trae como consecuencia que varios de los componentes del SNT propendan a cargarse en niveles cercanos a su capacidad nominal; e inclusive, en algunos casos, tengan que operar por sobre la capacidad nominal.

Para el caso del sistema eléctrico ecuatoriano, el SNI está compuesto, entre los elementos más relevantes por transformadores de potencia de niveles de voltaje de $(230 / 138 \mathrm{kV}, 230 / 69 \mathrm{kV}$ y $138 / 69 \mathrm{kV})$, repartidos en 19 subestaciones; que según la información publicada por el CONELEC en el Plan Maestro de Electrificación 2012-2021, están operando, actualmente, en niveles de cargabilidad que sobrepasan el $80 \%$ de su capacidad nominal; esto debido al incremento de la demanda o por inoperatividad de algunas centrales de generación.

Ante esta situación, es imperioso planificar, oportunamente, la expansión del SNT; y determinar los correspondientes requerimientos económicos y financieros. En este contexto, es menester de las instituciones el realizar estudios técnicos y económicos que permitan determinar la necesidad de nuevas líneas y subestaciones, el respectivo refuerzo de las instalaciones implementadas y eventualmente la necesidad o la conveniencia de sobrecargar los transformadores, por sobre sus límites operativos nominales, incluso con el riesgo de llegar al colapso (pérdida total del transformador). Este proceso implica, en unos casos, realizar inversiones cuantiosas para el sector eléctrico; o en su defecto disminuir la vida útil de los transformadores; con los correspondientes efectos económicos desfavorables [2].

\subsection{Cargabilidad de los transformadores de potencia}

Con el crecimiento de la demanda eléctrica, y con el fin de satisfacer la misma, se incrementa la carga de los transformadores por encima de su capacidad nominal, lo cual implica que se rebase el límite de temperatura de operación [3], es decir una temperatura superior a $140{ }^{\circ} \mathrm{C}$. lo que puede afectar al material dieléctrico del transformador [4]. Otro punto a considerar es la altitud sobre el nivel del mar (m s.n.m.) ya que al aumentar la altura disminuye la densidad del aire haciendo más difícil la evacuación del calor por lo cual incrementa la temperatura del transformador, esto depende del sistema de refrigeración que posee el mismo.

\subsection{Sistema de refrigeración}

Tipo OA: Sumergido en aceite, con enfriamiento natural. En estos transformadores, el aceite aislante circula por convección natural dentro de un tanque con paredes lisas, corrugadas o bien provistas de enfriadores tubulares o radiadores separables

Tipo OA/FA: Sumergido en aceite con enfriamiento propio y con enfriamiento de aire forzado. Este tipo de transformadores es básicamente una unidad OA a la cual se le han agregado ventiladores para aumentar la disipación del calor.

Tipo OA/FA/FOA: Sumergido en aceite con enfriamiento propio, con enfriamiento de aceite forzadoaire forzado. El régimen del transformador tipo OA, sumergido en aceite puede ser aumentado por el empleo combinado de bombas y ventiladores [5].

Tipo FOA: Sumergidos en aceite, con enfriamiento por aceite forzado con enfriadores de aire forzado. El aceite de estos transformadores es enfriado al hacerlo pasar por cambiadores de calor o radiadores de aire y aceite colocados fuera del tanque.

Tabla 1. Carga en base a temperatura [6].

\begin{tabular}{|c|c|c|c|}
\hline \multicolumn{4}{|c|}{$\begin{array}{l}\text { Carga en base a temperaturas } \\
\text { (Temp. ambiente y promedio de elevación de temperatura } \\
\text { menor de los valores línites, para una aproximación rápida } \\
\text { rango de temperatura ambiente de } 0^{\circ} \mathrm{C} \text { a } 50^{\circ} \mathrm{C} \text { ) }\end{array}$} \\
\hline Tipo de re & frigeración & $\begin{array}{l}\text { \% de capacidad } \\
\text { Disminución de carga } \\
\text { por cada grado de } \\
\text { elevación de } \\
\text { temperatura }\end{array}$ & $\begin{array}{l}\text { Incremento de carga } \\
\text { por cada grado de } \\
\text { disminución de } \\
\text { temperatura }\end{array}$ \\
\hline Auto refrigerado & $\mathrm{OA}$ & 1.5 & 1 \\
\hline Por agua & OW & 1.5 & 1 \\
\hline Aire & $\mathrm{OA} / \mathrm{FA}$ & & \\
\hline forzado & $\mathrm{OA} / \mathrm{FA} / \mathrm{FA}$ & 1 & 0.75 \\
\hline Aire & FOA, FOW y & & \\
\hline forzado & $\mathrm{OA} / \mathrm{FOA} / \mathrm{FOA}$ & 1 & 0.75 \\
\hline
\end{tabular}

\subsection{Normativa}

- Norma ANSI/IEEE C57.92.1981

Esta norma se basa principalmente en el envejecimiento del aislamiento del bobinado del transformador, las predicciones de la expectativa de la vida de un transformador, están basadas en una tabla donde se muestran las curvas de expectativa producidas por las variaciones de temperatura, pero no toman en cuenta el deterioro de las juntas, la oxidación de los tanques, etc., que también afectan a la vida útil del transformador [7].

\section{- Norma IEC 60076-7}

La norma habla de las especificaciones de los regímenes de carga de los transformadores, y el punto más 


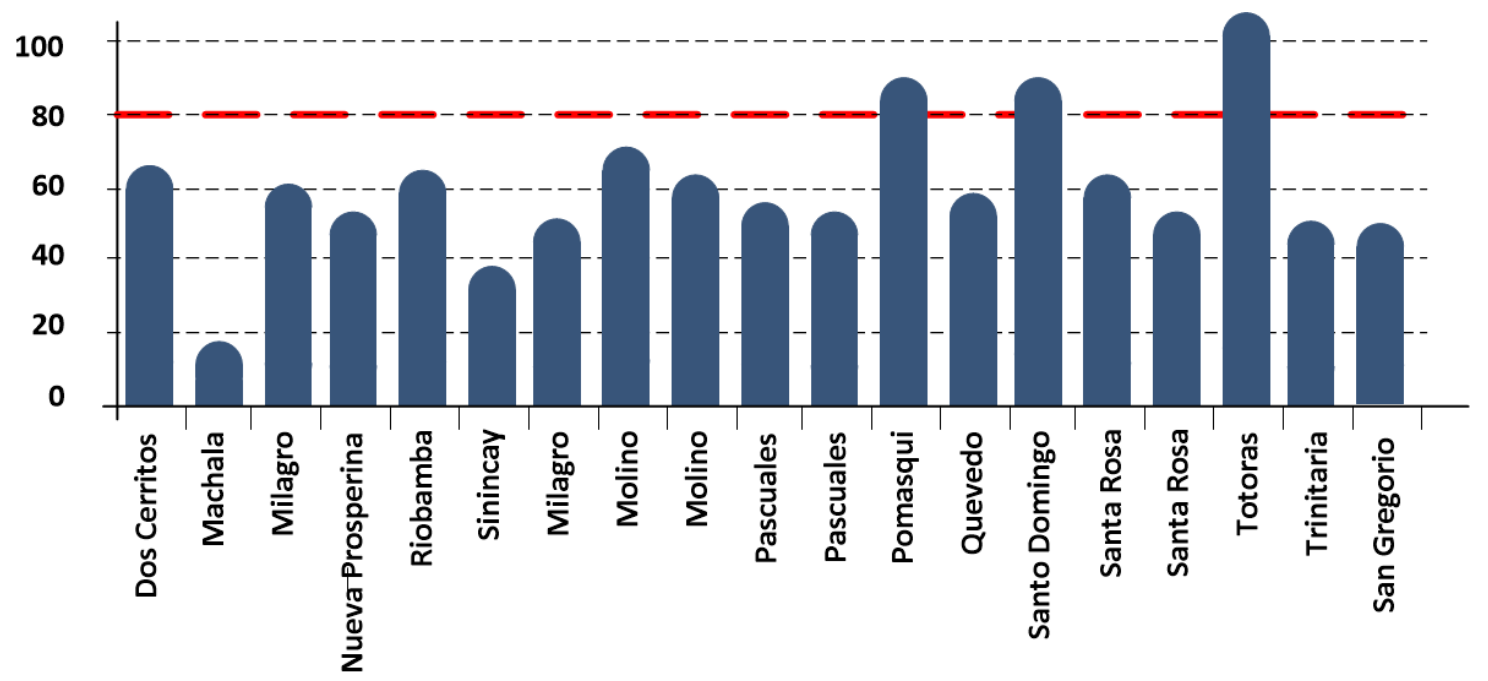

Figura 1. Cargabilidad Elementos SNT - Transformadores del SNT (230/138 y 230/69) kV [1].

caliente del exceso de temperatura y del envejecimiento térmico relativo, por lo que prevé los regímenes de carga a evaluar [8].

Proporciona recomendaciones para el régimen de carga mayor al asignado para su valor asignado, y una guía para las condiciones adecuadas de carga para las nuevas instalaciones [9].

\section{Modelamiento}

Para el desarrollo del programa de modelamiento y simulación, y luego de realizar el análisis de las normativas existentes, obtenemos los datos de la placa de características la cual posee un indicativo de las características eléctricas del transformador [7], como se describe a continuación:

- Tipo de transformador.

- Número de esta norma.

- Nombre del fabricante.

- Número de serie del fabricante.

- Año de fabricación.

- Número de fases.

- Potencia nominal (en KVA o MVA).

- Frecuencia asignada (en $\mathrm{Hz}$ ).

- Tensiones nominales (en V o KV).

- Corrientes nominales (en A o KA).

- Símbolo de acoplamiento.

- Impedancia de cortocircuito (expresado en \%).
- Tipo de sistema de refrigeración.

- Masa total.

- Masa del aceite aislante.

Partiendo de estos datos, se inicia con el desarrollo del modelo computacional en el cual se considerarán los principales parámetros asociados con la cargabilidad de los transformadores, todo esto sustentado en normas, para la formulación técnica, mediante el cual se seleccionará el modelo que mejor se acopla a nuestras necesidades de estudio [10].

Con la selección del modelo matemático, se desarrolla, una simulación dentro de una plataforma de software de modelado matemático como lo es MATLAB ${ }^{\circledR}$ [11].

Se evalúan todos los parámetros y características de una situación particular (como lo es la cargabilidad de los transformadores de potencia), y se analizarán varios de los datos y resultados obtenidos; delimitando el problema, formulando una hipótesis, y con los datos recolectados se redactará un informe para saber cuál es el punto óptimo de cargabilidad del transformador en estudio.

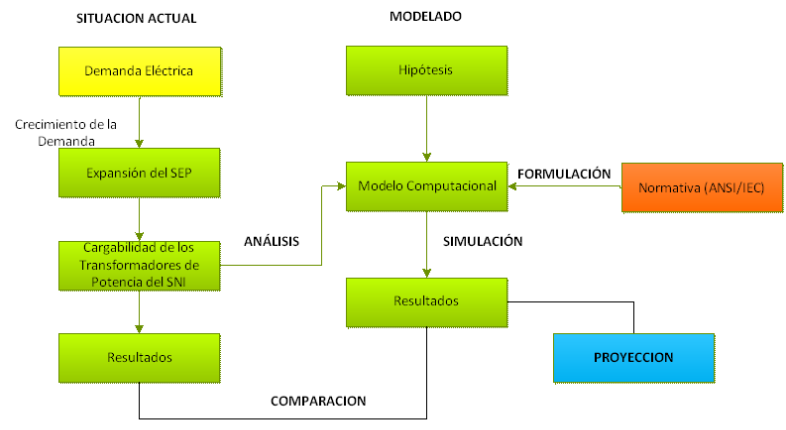

Figura 2. Modelado de la cargabilidad de los transformadores de potencia del SNI (propuesta de estudio). 


\subsection{Estructura del modelo computacional}

El contenido del modelo computacional se basa en la formulación técnico-matemática obtenida de las ecuaciones de la normativa y estándares que rigen la cargabilidad de los transformadores de potencia.

El objetivo es utilizar las ecuaciones que están documentadas en las normas, y trasladarlas a un algoritmo matemático que abarca un software, para poder ingresar los datos obtenidos de la placa de características, es decir con estos datos se puede determinar el nivel óptimo de cargabilidad para el transformador, para que con los resultados obtenidos se pueda realizar un análisis técnico-económico y bajo parámetros técnicos se pueda realizar una proyección del estado de la vida útil del transformador [12] [13].

La versión final del modelo computacional permite al usuario realizar una simulación y obtener resultados del estado de cargabilidad del transformador en análisis, esto permite obtener reportes y realizar comparaciones de estados con el uso de otro tipo de transformador ya que el programa permite cambiar y variar datos.

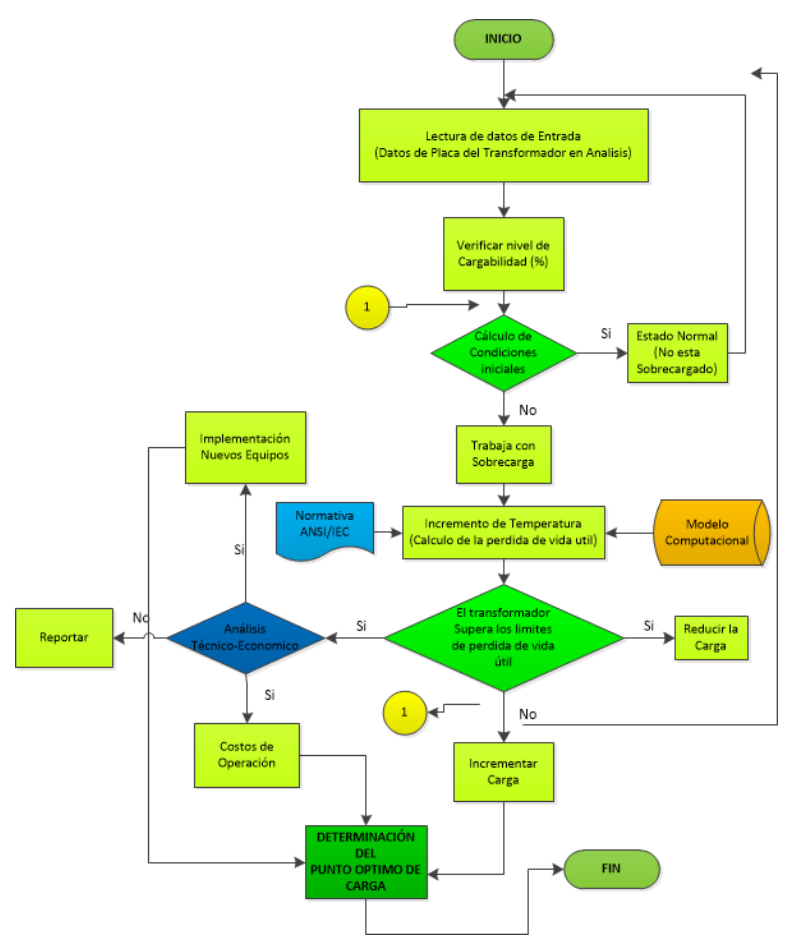

Figura 3. Flujograma del algoritmo para el modelo computacional [14].

\subsection{Formulación}

El principal parámetro involucrado en la formulación matemática es el correspondiente a las variaciones de la temperatura que se presentan en el transformador.
La variación de temperatura depende de la constante del transformador, que a su vez depende de la capacidad de calor del transformador de tiempo térmico global, es decir, la masa del núcleo, bobinas, y el aceite, y la tasa de transferencia de calor fuera del transformador [15]. Como una función del tiempo, el cambio de temperatura se modela como una respuesta exponencial de primer orden desde el estado de temperatura inicial para el estado temperatura final como se indica en la siguiente ecuación [13] y [16].

$$
\Delta \theta T_{o i l}=\left[\Delta \theta o_{u}-\Delta \theta o_{i}\right]\left[1-\exp ^{\frac{-t}{T}} T_{o}\right]+\Delta \theta_{o i}
$$

Cuando $\Delta \theta o_{i}$ es el aumento de la temperatura inicial, $\Delta \theta o_{u}$ es el aumento de la temperatura final, $T_{t o}$ es la parte superior de aceite constante de tiempo, $t$ es el tiempo hace referencia a la hora del cambio de carga y $\Delta \theta_{\text {Toil }}$ es el aumento de la temperatura de aceite superior sobre variable de la temperatura ambiente [17].

Como último procedimiento para determinar la temperatura del punto más caliente del transformador, se calcula mediante la adición de la temperatura ambiente a la parte superior del aumento de la temperatura del aceite y para hallar el aumento de la temperatura en el punto caliente, entonces usamos:

$$
\theta_{H}=\theta_{A}+\Delta \theta_{H}+\Delta \theta_{\text {Toil }}
$$

Donde: $\theta_{H}$ es la temperatura del punto más caliente y $\theta_{A}$ es la temperatura ambiente [18].

En la guía ANSI/IEEE [6] se establece una selección en base a criterios técnicos y propia estimación de la posibilidad de vida útil. En esta guía, 180000 h (20,6 años), se utilizan como una vida normal. Se supone que el deterioro del aislamiento puede ser modelado como una cantidad por unidad con la siguiente ecuación [3]:

$$
\text { Pérdida de vida útil }=A\left[B_{\theta H}+273\right] e
$$

Donde $A$ es una constante modificada basada en la temperatura establecida para determinar la vida útil y la unidad $B$ es la tasa de envejecimiento que afecta al transformador [19]. Para una temperatura de referencia de $140^{\circ} \mathrm{C}$, la ecuación para envejecimiento acelerado es [4].

\subsection{Determinación del nivel óptimo de carga- bilidad}

Todo este análisis y simulación en el software inducirá a realizar los análisis pertinentes para determinar el nivel óptimo de la carga para el transformador de la Subestación Pomasqui desde el punto de vista técnico-económico ya que éste presenta un alto índice de cargabilidad sobre el $80 \%$ según lo publicado en 
el Plan Maestro de Electrificación 2012-2021 del libro publicado por el CONELEC [1].

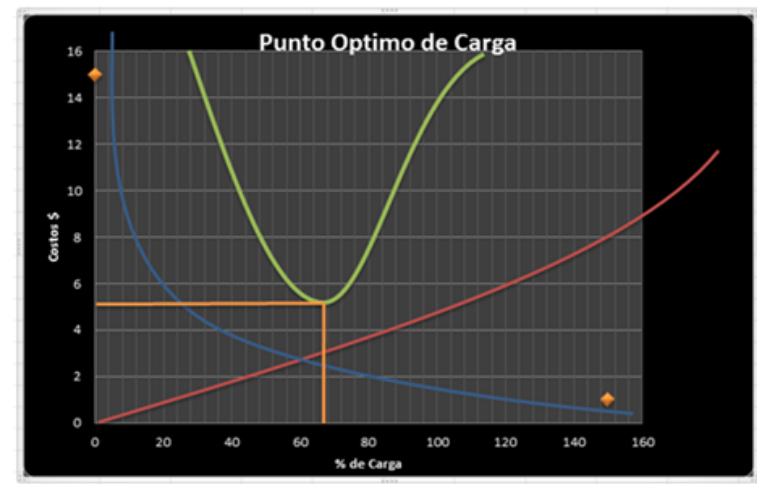

Figura 4. Determinación del punto óptimo de cargabilidad (objetivo del modelo computacional).

Donde:

- Línea azul: Representa los costos de inversión para el Sistema Eléctrico Ecuatoriano.

- Línea Roja: Representa la perdida de vida útil del transformador.

- Línea verde: Sumatoria de los resultados, es decir, esto determinará el punto óptimo económico de carga para el transformador.

\subsection{Contribución del modelamiento}

Definir hasta qué nivel se puede sobrecargar un transformador actualmente en operación para satisfacer la demanda, sin necesidad de realizar nuevas inversiones y determinar desde que nivel de sobrecarga es recomendable en términos técnicos-económicos instalar un transformador adicional para evitar la sobrecarga del actualmente en operación.

\section{Conclusiones}

El valor agregado de desarrollar un modelo computacional para determinar el nivel óptimo de cargabilidad de los transformadores del Sistema Eléctrico Ecuatoriano, radica en que con la aplicación del modelo y la evaluación de resultados se establecerá, con sustentos técnicos y económicos la posibilidad de diferir inversiones sobrecargando el transformador actualmente en servicio; o realizar inversiones para la instalación de un nuevo transformador adicional, opción que al momento en el país no se la tiene disponible.

Con el modelo computacional se pueden correr varios escenarios de operación del transformador de la Subestación Pomasqui 230/138 KV, para con los resultados determinar el nivel óptimo de operación que se sustenta en la evaluación técnico-económica que contiene la formulación del modelo.

El evaluar los resultados permite establecer recomendaciones y proyecciones del estado de los equipos para un futuro dentro del Sistema Nacional Interconectado [20].

\section{Referencias}

[1] CONELEC, "Plan maestro de electrificación $2012, " 2012$.

[2] L. L. Grigsby, Electric Power Generation Transmission and Distribution, 3rd ed., ser. The Electric Power Engineering Handbook. Taylor \& Francis, 2012.

[3] K. Najdenkoski, G. Rafajlovski, and V. Dimcev, "Thermal aging of distribution transformers according to ieee and iec standards," in Power Engineering Society General Meeting, 200\%. IEEE. IEEE, 2007, pp. 1-5.

[4] I. 60076-7, Loading guide for Oil-immersed Power Transformers, Std., 2005.

[5] L. Pierce, "Predicting liquid filled transformer loading capability," IEEE Transactions on Industry Applications, vol. 30, no. 1, pp. 170-178, 1994.

[6] ANSI/IEEE, Distribution, power and regulating transformers, Std., 1994.

[7] F. J. Yébenes Cabrejas, "Gestión de la cargabilidad de transformadores de potencia," Proyecto de fin de carrera, Universidad Carlos III de Madrid, Madrid, España, 2009.

[8] "IEEE guide for determination of maximum winding temperature rise in liquid-filled transformers," IEEE Std 1538-2000, 2000.

[9] P. Code, International Standard IEC 60076-7, Std.

[10] O. Ramírez and S. Fernández, "Introducción de un modelo térmico para el diagnóstico en tiempo real de transformadores," La Habana, Cuba, 2000.

[11] D. Kalluri, Electromagnetic Waves, Materials, and Computation With MATLAB. Taylor \& Francis, 2011.

[12] W. Tang, Q. Wu, and Z. Richardson, "A simplified transformer thermal model based on thermalelectric analogy," IEEE Transactions on Power Delivery, vol. 19, no. 3, pp. 1112-1119, 2004. 
[13] B. Lesieutre, W. Hagman, and J. Kirtley, J.L., "An improved transformer top oil temperature model for use in an on-line monitoring and diagnostic system," IEEE Transactions on Power Delivery, vol. 12, no. 1, pp. 249-256, 1997.

[14] T. Shamsodin, G. Ahmad, F. Issouf, and T. Hamed, "Modeling and simulation of transformer loading capability and hot spot temperature under harmonic conditions,", Electric Power Systems Research, vol. 86, pp. 68-75, May 2012.

[15] IEC-61378-1, Transformers for industrial applications, Std., 1997.

[16] D. J. Tylavsky, Q. He, J. Si, G. A. McCulla, and J. R. Hunt, "Transformer top-oil temperature modeling and simulation," IEEE Transactions on Industry Applications, vol. 36, no. 5, pp. 1219$1225,2000$.

[17] A. Emanuel and X. Wang, "Estimation of loss of life of power transformers supplying nonlinear loads," IEEE Transactions on Power Apparatus and Systems, vol. PAS-104, no. 3, pp. 628-636, 1985.

[18] L. Pierce, "Predicting liquid filled transformer loading capability," in Petroleum and Chemical Industry Conference, 1992, Record of Conference Papers., Industry Applications Society 39th Annual, 1992, pp. 197-207.

[19] Y.-C. Huang, H.-T. Yang, and C.-L. Huang, "Developing a new transformer fault diagnosis system through evolutionary fuzzy logic," IEEE Transactions on Power Delivery, vol. 12, no. 2, pp. 761-767, 1997.

[20] O. Gouda, G. Amer, and W. Salem, "Predicting transformer temperature rise and loss of life in the presence of harmonic load currents," Ain Shams Engineering Journal, vol. 3, no. 2, pp. 113-121, 2012. 